\title{
Tratamento de Trombose Tardia de Stent Farmacológico com Cateter Extrator de Trombo
}

\author{
Marcus Ernesto Sampaio Lacativa', Leonardo Furtado de Oliveira ${ }^{1}$, Rodrigo de Franco Cardoso', \\ João Luiz Frighetto ${ }^{2}$, Rodolfo de Franco Cardoso ${ }^{3}$, Julio Eduardo Campos ${ }^{1,4}$, \\ Francisco Cabral Cardoso', Luiz Paulo Rebello Alves ${ }^{1}$
}

\section{RESUMO}

Relatamos o caso de um paciente de 69 anos com trombose tardia de stent farmacológico, admitido com infarto agudo do miocárdio anterior, tratado com sucesso com o uso de cateter de aspiração de trombos (Pronto ${ }^{\mathrm{TM}} \mathrm{V} 3$ ).

DESCRITORES: Contenedores. Trombose coronária. Trombectomia.

\section{SUMMARY}

Treatment of Late Thrombosis of Drug-eluting Stent Using a Thrombus Extraction Catheter

We report on the case of a 69-year-old patient with late thrombosis of a drug-eluting stent, admitted to hospital with anterior AMI, who was successfully treated using a thrombus extraction catheter (Pronto ${ }^{\mathrm{TM}} \mathrm{V} 3$ ).

DESCRIPTORS: Stents. Coronary thrombosis. Thrombectomy.
A tualmente, existe uma grande preocupação mundial com o risco de trombose tardia em stents farmacológicos, dadas as suas graves implicações clínicas ${ }^{1}$. A sua alta letalidade, chegando a $45 \%{ }^{2}$, torna sua prevenção algo a ser buscado de forma obstinada e retrata a dificuldade de tratamento desta entidade.

O principal preditor independente da trombose tardia é a descontinuação dos antiplaquetários (IR 89,78, $\mathrm{p}<0,001)^{2}$, podendo os índices de trombose de stent superar $25 \%$ se o clopidogrel for suspenso antes de 30 dias após o implante do stent ${ }^{3}$. Os grandes desafios no tratamento são a quantidade de trombos e o no reflow. Apesar de todo o arsenal terapêutico à disposição atualmente (trombolíticos, inibidores de glicoproteína Ilb/IIla, filtros e extratores de trombo), as taxas de infarto agudo do miocárdio (IAM) e mortalidade permanecem elevadas ${ }^{4}$.

A remoção do trombo coronariano parece reduzir o tamanho do infarto e melhorar o fluxo coronariano

\footnotetext{
1 HEMOCOR - Serviço de Hemodinâmica e Angiocardiografia de Jacarepaguá - Rio de Janeiro, RJ.

2 HFAG - Hospital da Força Aérea do Galeão.

3 HUCFF - Hospital Universitário Clementino Fraga Filho.

4 HCE - Hospital Central do Exército.

Correspondência: Marcus Ernesto Sampaio Lacativa. R. Bacairis, 499 2o andar - Taquara - Jacarepaguá - Rio de Janeiro - RJ - CEP: 22730-120 Tel.: (21) 2423-3621 - Fax: (21) 2423-4047•E-mail: mlacativa@ig.com.br Recebido em: 27/2/2007 • Aceito em: 26/3/2007
}

em pacientes com trombose de stent. O cateter Pronto ${ }^{\mathrm{TM}}$ V3 (Vascular Solutions) é um cateter de troca rápida com um duplo lúmen, ponta atraumática e hidrofílica, com um grande lúmen para extração de trombos, sendo de fácil e rápido manuseio. Os dados preliminares do DEAR-MI mostraram eficácia e segurança com o seu uso $^{5}$ e, alguns casos, com excelente resultado; aspiração de trombos macroscópicos já foi descrita na literatura ${ }^{6-8}$, inclusive de pacientes com trombose tardia de stent $^{6}$.

\section{RELATO DO CASO}

Paciente masculino de 69 anos, com história de angioplastia coronariana com implante de stent com eluição de paclitaxel - Taxus (Boston Scientific) - 2,75x $28 \mathrm{~mm}$, em segmento proximal da artéria descendente anterior, quatro meses antes do evento, quando foi atendido em fase subaguda de IAM anterior, apresentando-se agora com precordialgia importante, iniciada há 30 minutos, e com supradesnivelamento do segmento ST de V1 a V4.

O paciente relatava melena 10 dias antes à internação, sendo investigado com endoscopia digestiva, que não mostrou alterações. Foi solicitada colonoscopia pelo médico assistente e suspenso o uso de antiplaquetários (ácido acetilsalicílico e clopidogrel), cinco dias antes de sua internação.

À chegada ao hospital, apresentava-se sudoreico, 
pálido, referindo precordialgia 8+/10, com alteração do eletrocardiograma (ECG) em parede anterior (Figura 1).

A coronariografia revelou trombose de stent proximal em artéria descendente anterior, com fluxo TIMI 0 , sem demais lesões coronarianas significativas (Figura 2). Administrados $600 \mathrm{mg}$ de clopidogrel visando à realização de angioplastia primária deste vaso.

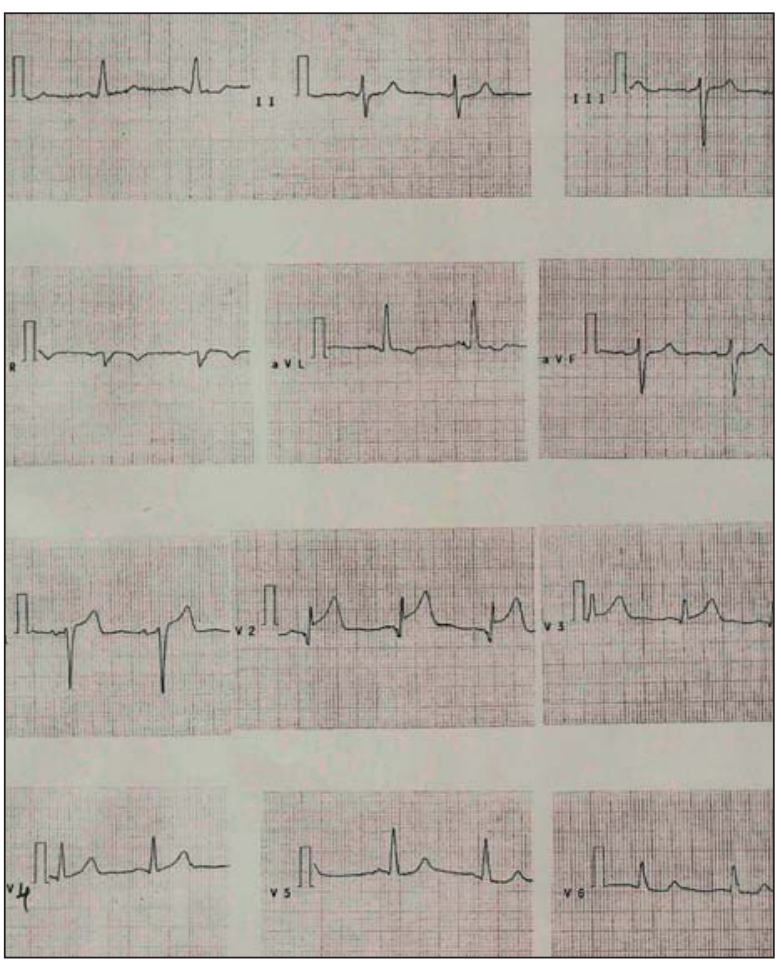

Figura 1 - ECG de admissão.

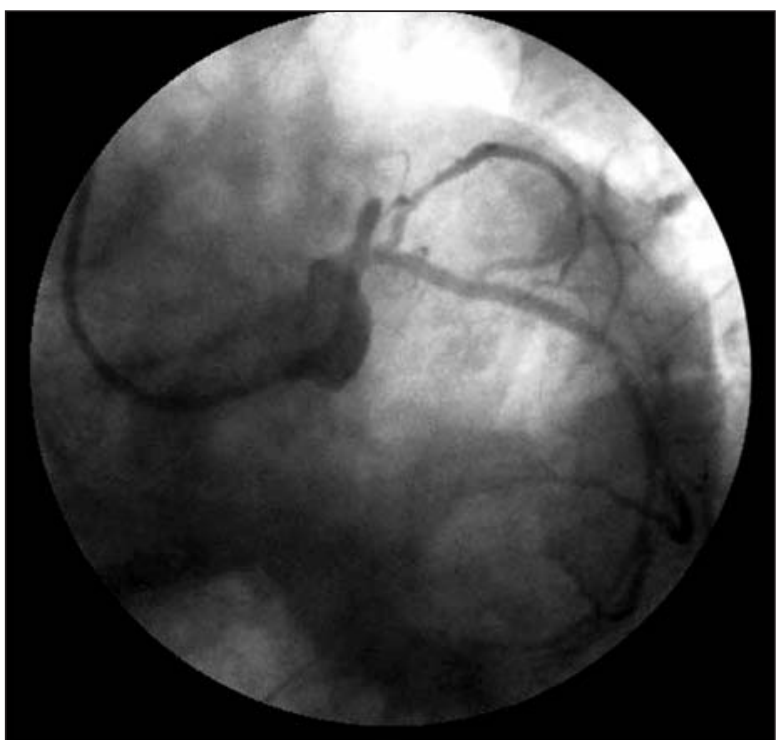

Figura 2 - Oclusão proximal de DA.
Cateterizada a artéria coronária esquerda com cateter-guia JL4 6F, passamos corda-guia 0,014 para o leito distal da artéria descendente anterior, seguida da passagem de cateter extrator de trombos Pronto V3, sendo aspirados dois grandes trombos (Figura 3). Após o procedimento, obtivemos fim da precordialgia, resolução do supradesnivelamento de ST ao monitor e aparecimento de arritmias de reperfusão (extra-sístoles e ritmo idioventricular acelerado)

O resultado angiográfico final mostrava fluxo TIMI 3 com Blush 3, sem embolização distal, não havendo necessidade de implante de outro stent (Figura 4). Não foi usado inibidor de glicoproteína IIb/IIla.

O paciente apresentou boa evolução clínica, evoluindo sem precordialgia e com resolução do supradesnivelamento de ST ao ECG (Figura 5), recebendo alta após $72 \mathrm{~h}$.

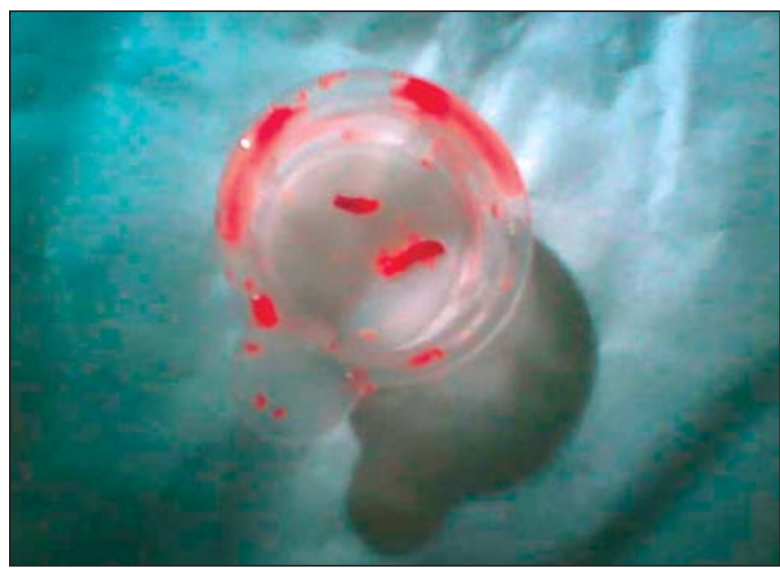

Figura 3 - Trombos aspirados com cateter Pronto.

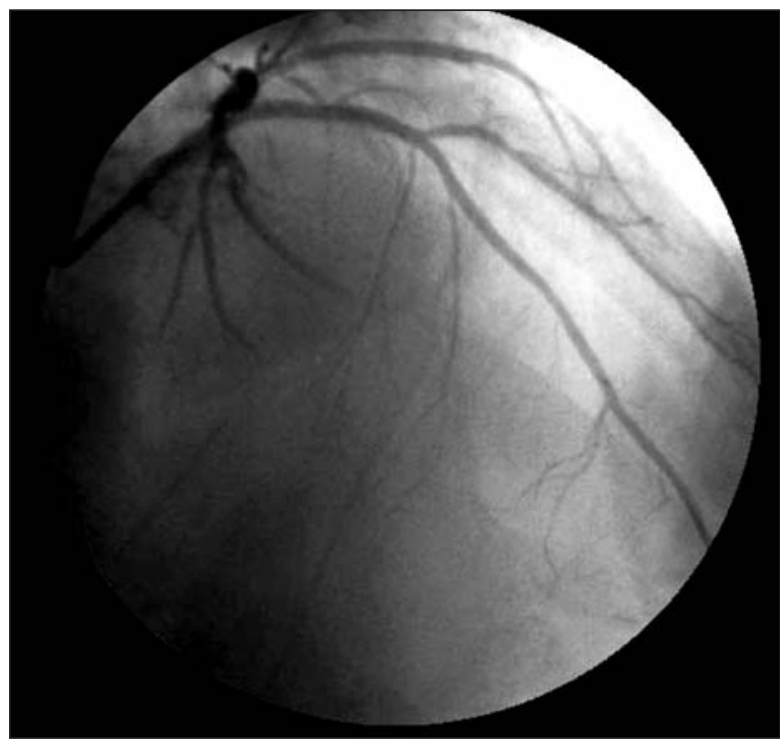

Figura 4 - Resultado após aspiração de trombos. 


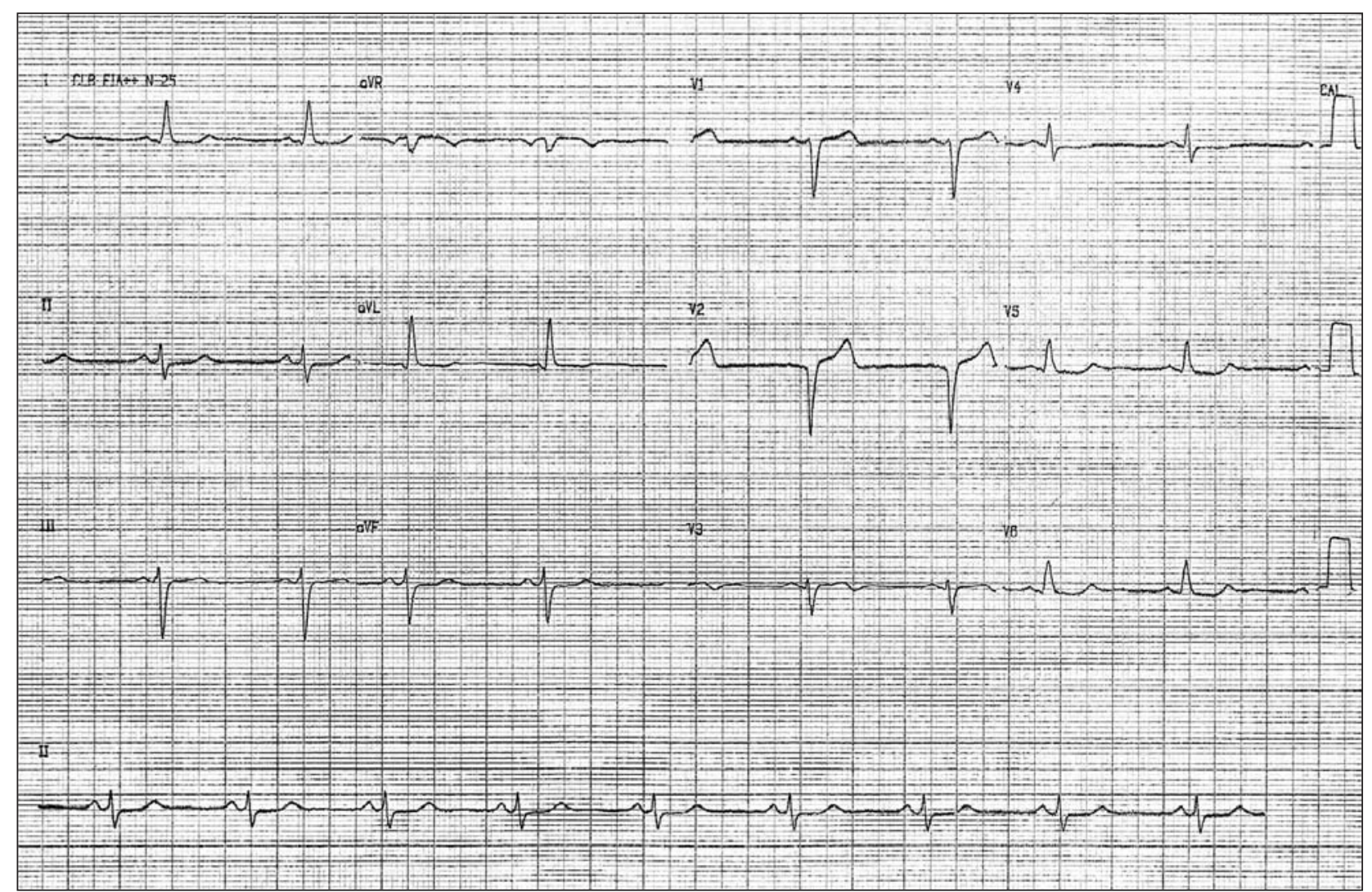

Figura 5 - ECG realizado imediatamente após o procedimento.

\section{DISCUSSÃO}

Em nosso serviço, já havíamos usado o cateter Pronto em casos de IAM com grande carga trombótica, mas este foi o primeiro caso de uso em trombose de stent. A facilidade no manuseio do cateter e a rapidez em seu uso nos motivou a usá-lo quando nos deparamos com o caso. O resultado extremamente satisfatório nos impressionou, já que em tais casos a abordagem com cateter-balão mostra altos índices de no reflow e a obtenção de Blush 3, muitas vezes, é extremamente difícil, devido à embolização distal para a microvasculatura. A restauração do fluxo só é obtida em $48 \%$ dos casos $^{9}$ e a embolização distal ocorre em $15 \%$ dos casos e tem grande influência prognóstica, sendo fator preditivo independente de mortalidade em $5 \operatorname{anos}^{10}$. O tratamento com trombolítico também não se mostrou efetivo em casos de trombose de stent ${ }^{9}$. A resolutividade no caso descrito foi extremamente rápida, o que é importante, não sendo necessário uso de outro stent ou de inibidor de glicoproteína Ilb/IIla, reduzindo o custo final do procedimento.

Os resultados do estudo AIMI com o AngioJet foram extremamente desanimadores ao mostrar um aumento na mortalidade e no tamanho do infarto nos pacientes tratados com o Angiojet ${ }^{11}$.

No DEAR-MI, não foram incluídos pacientes com trombose de stent, mas os resultados, com 60 pacientes com IAM randomizados a receberem tratamento com angioplastia ou com aspiração de trombos com o cateter Pronto seguida de angioplastia ${ }^{5}$, mostraram uma melhora do blush miocárdico (78,6\% Pronto vs. 38,5\% controle, $p<0,01)$ e maior redução do supradesnivelamento do segmento ST $(64,2 \%$ Pronto vs. $35,5 \%$ controle, $\mathrm{p}<0,05)$, sem diferença quanto aos eventos cardíacos maiores intra-hospitalares $(3,4 \%$ Pronto vs. $3,2 \%$ controle, $\mathrm{p}=\mathrm{ns}$ ).

A nova geração do cateter Pronto - o Pronto V3 - possui uma porção distal hidrofílica, o que facilitou bastante o seu uso com cateter-guia $6 \mathrm{~F}$, diminuindo o tempo de procedimento, já que não houve necessidade de troca do introdutor arterial e cateter-guia, e as complicações vasculares pelo acesso.

\section{CONCLUSÃO}

Com o aumento progressivo na utilização de stents farmacológicos e aumento do número absoluto de angioplastias, a tendência é uma ocorrência maior em números absolutos de trombose tardia de stent. O tratamento percutâneo usado nos dias de hoje é subótimo. O cateter Pronto V3, além de fácil manuseio, pode acelerar a obtenção de um resultado satisfatório. $\mathrm{O}$ impressionante resultado que obtivemos sugere que 
este cateter deva ser avaliado em estudos maiores para o tratamento de pacientes com trombose de stent. A prevenção, contudo, continua sendo nossa grande arma. As diretrizes norte-americanas e européias já sugerem a antiagregação com dois antiplaquetários por 12 meses, a menos que haja um alto risco de sangramento. Essa orientação, contudo, não foi acompanhada pelas companhias fabricantes dos stents farmacológicos ${ }^{12}$.

\section{REFERÊNCIAS BIBLIOGRÁFICAS}

1. Moussa I, Di Mario C, Reimers B, Akiyama T, Tobis J, Colombo A. Subacute stent thrombosis in the era of intravascular ultrasound-guided coronary stenting without anticoagulation: frequency, predictors and clinical outcome. J Am Coll Cardiol. 1997;29(1):6-12.

2. lakovou I, Schmidt T, Bonizzoni E, Ge L, Sangiorgi GM, Stankovic G, et al. Incidence, predictors, and outcome of thrombosis after successful implantation of drug-eluting stents. JAMA. 2005;293(17):2126-30.

3. Jeremias A, Sylvia B, Bridges J, Kirtane AJ, Bigelow B, Pinto DS, et al. Stent thrombosis after successful sirolimus-eluting stent implantation. Circulation. 2004;109(16):1930-2.

4. Mishkel GJ, Moore AL, Markwell S, Shelton MC, Shelton ME. Long-term outcomes after management of restenosis or thrombosis of drug-eluting stents. J Am Coll Cardiol. 2007; 49(2):181-4.

5. Colombo P, Silva P, Delgado R, Bigi R, Bossi I, Salvade P, et al. Thrombus aspiration with the Pronto extraction cathe- ter before standard percutaneous coronary intervention improves myocardial reperfusion: preliminary results of a prospective randomized study. Am J Cardiol. 2004;94:12E.

6. Siddiqui DS, Choi CJ, Tsimikas S, Mahmud E. Successful utilization of a novel aspiration thrombectomy catheter (Pronto) for the treatment of patients with stent thrombosis. Catheter Cardiovasc Interv. 2006;67(6):894-9.

7. Cardoso RF, Lacativa MES, Campos JE, Frighetto JL, Oliveira LF, Alves LPR, et al. Utilização de cateter extrator de trombo em infarto agudo do miocárdio com grande carga trombótica. Rev Bras Cardiol Invas. 2005;13(2):121-3.

8. Santos MA, Fonseca CGB, Marasca M, Godoy MF. Sistema de trombectomia com aspiração manual durante angioplastia primária no infarto agudo do miocárdio. Rev Bras Cardiol Invas. 2005;13(2):129-30.

9. Hasdai D, Garratt KN, Holmes DR Jr, Berger PB, Schwartz RS, Bell MR. Coronary angioplasty and intracoronary thrombolysis are of limited efficacy in resolving early intracoronary stent thrombosis. J Am Coll Cardiol. 1996;28(2):361-7.

10. Gick M, Jander N, Bestehorn HP, Kienzle RP, Ferenc M, Werner K, et al. Randomized evaluation of the effects of filter-based distal protection on myocardial perfusion and infarct size after primary percutaneous catheter intervention in myocardial infarction with and without ST-segment elevation. Circulation 2005;112(10):1462-9.

11. O'Neill WW, Dixon SR, Grines CL. The year in interventional cardiology. J Am Coll Cardiol. 2005;45(7):1117-34.

12. Shuchman M. Trading restenosis for thrombosis? New questions about drug-eluting stents. N Engl J Med. 2006;355(19): 1949-52. 